\title{
Permanent magnetic system design for the wall-climbing robot
}

doi:10.1533.abbi.2006.0024

\author{
W. Shen ${ }^{1}$, J. Gu ${ }^{1,2}$ and Y. Shen ${ }^{3}$ \\ ${ }^{1}$ Electrical and Computer Engineering, Dalhousie University, Halifax, NS Canada B37 2 X4 \\ ${ }^{2}$ School of Control Science and Engineering, Shandong University, People's Republic of China 250100 \\ ${ }^{3}$ College of Science, Three Gorges University, Yichang, Hubei, 443002, China
}

\begin{abstract}
This paper presents the design and analysis of the permanent magnetic system for a wallclimbing robot with permanent magnetic tracks. Based on the behaviour of gecko lizards, the architecture of the robot was designed and built, including the structure of the adhesion mechanism, the mechanical architecture and the anti-toppling mechanism. The permanent magnetic adhesion mechanism and the tracked locomotion mechanism were employed in this kind of wall-climbing robot. Through static and dynamic force analysis of the robot under different situations, design requirements for the adhesion mechanism were derived. Two different types of structures were put forward for the permanent magnetic units and are further discussed in this paper. These two types of structures are also analysed in detail. In addition, a finite-element method was used to verify the results of magnetic units. Finally, two wall-climbing robots, equipped with different magnetic systems described previously, are explained and their applications are discussed in this paper.
\end{abstract}

Key words: Wall-climbing robot, magnetic adhesion mechanism, tracked locomotion mechanism, antitoppling system.

\section{INTRODUCTION}

In the robotics field, there exist many different kinds of robots, for example, under-water robots, flying robots and mobile robots. The design of most of these robots is motivated by the behaviour of animals. The same can be said for wall-climbing robots. When people observed gecko lizards, flies and other animals that can walk on a vertical wall, they were inspired to develop a device that can move in a similar fashion on a vertical wall. On the basis of research conducted on animal behaviour, different adhesion mechanisms, such as a vacuum sucker pad, a magnetic wheel and dry adhesion, for wall-climbing robots were developed. Recent studies have proved that it is the physical structure of a gecko's feet that allows it to stick to walls. In 2000, biologists and other researchers proved that the tree-like hair structure on the soles of the geckos' feet helped them stick to vertical walls. The strength of this attraction was 10 times more than what they had assumed. Gecko's unique

Corresponding Author:

W. Shen

Department of Electrical and Computer Engineering

1360 Barrington Street, Sexton Campus

Dalhousie University, Halifax, NS B3J 121

Canada

Email: Weimin.Shen@dal.ca feet are not the result of special chemicals or treatments, they never get dirty or leave a residue, they can stick to any material except Teflon and they can attach and detach their feet with little effort (Autumn et al. 2000). Sitti and Fearing (2003) proposed that this kind of foot will be used in future to develop wall-climbing robots.

The wall-climbing robot's design, which is different from that of a mobile robot, has two critical issues. First is the special adhesion mechanism needed to support the robot on the wall safely. The other is the locomotion mechanism to allow the robot to move on the wall efficiently.

Four kinds of locomotion mechanisms are commonly used by wall-climbing robots, including a wheeled mechanism (Schmid et al. 1998), a tracked mechanism (Wang et al. 1999), a legged mechanism (Luk et al. 1999) and locomotion based on arms and grippers (White et al. (1998)). In general, four types of adhesion mechanisms are adopted by wall-climbing robots; these are magnetic adhesion, vacuum sucker pads, propellers (Nishi and Miyagi 1994) and dry adhesion using nanofabrication techniques (Sitti and Fearing 2003). Different adhesion mechanisms and different locomotion mechanisms can be combined to form different types of wall-climbing robots.

Because wall-climbing robots can extend a person's workspace, increase operational efficiency and even replace humans in hazardous tasks, for example, the cleaning of high-rise buildings, they have recently become very 


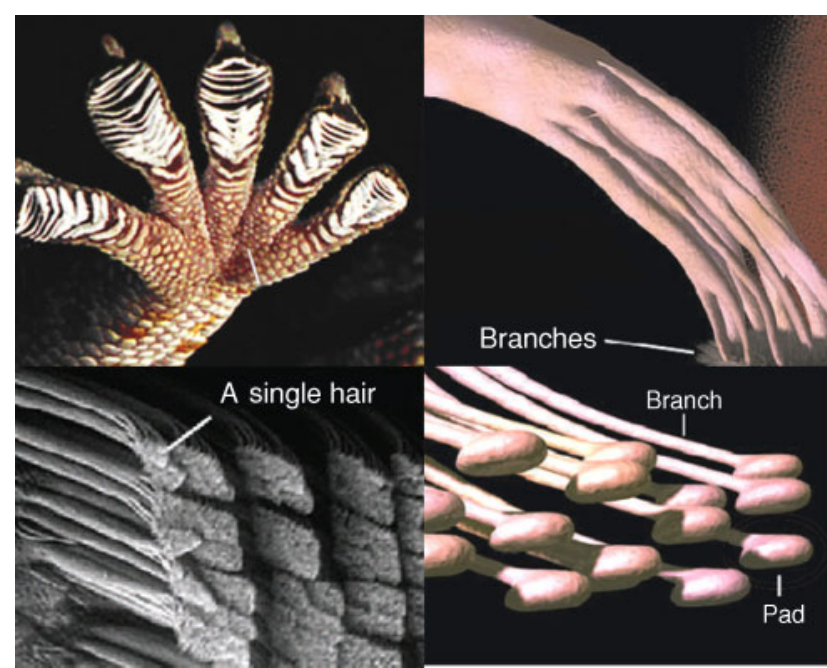

Figure 1 The structure of a gecko's foot.

popular and attractive. Currently, there are several industrial applications where wall-climbing robots are employed, such as the inspection and maintenance of storage tanks in nuclear power plants and petrochemical enterprises (Briones et al. 1994; Savall et al. 1999), ship hull welding and cleaning (Griecho et al. 1998), fire fighting (Amano et al. 2001) and cleaning of high-rise buildings (Zhang et al. 2004a, 2004b). The present study aims to design a wall-climbing robot that is able to inspect oil tanks with non-destructive testing (NDT) tools.

The first wall-climbing robot dates back to the last century, specifically the 1960s, and was developed by Nishi et al. (1986) in Japan. This wall-climbing robot had a large volume, was very heavy, and used a single vacuum-sucking cup. Later, during the 1990 s, wall-climbing robots were developed rapidly. More recently, various wall-climbing robots have been developed for wall cleaning, inspection, maintenance etc. A family of sky cleaner robots, which are totally actuated by pneumatic cylinders and which adhere to glass walls with vacuum suckers, is presented in Zhang et al. (2004a, 2004b). This robot is lightweight and dexterous due to the pneumatic actuators, but it still needs a supporting system to secure its safety. A miniature wallclimbing robot was developed by Xiao et al. (2003) and Tummala et al. (2002). This climbing robot is supported by two suction feet that can grip both smooth and nonporous surfaces. A three-limbed robot capable of climbing vertical natural terrain is discussed in Bretl et al. (2003). This robot can climb real, vertical natural terrain, and has applications in military and civilian domains, such as search, rescue, reconnaissance and planetary exploration. A quadruped walking and climbing robot for NDT was developed by Kang et al. (2003). This robot, called 'MRWALL SPECT III', can walk on plane surfaces, as well as climb walls with the help of suction pads, and carry an ultrasonic NDT tool for inspection of the large surface of industrial utilities. In Almonacid et al. (2003), the authors present a novel application of the Stewart-Gough parallel platform as a climbing robot and it climbs high structures through unknown spatial trajectories, such as palm trunks, tubes, etc. In Abderrahim et al. (1999), the ROMA robot, a specially developed self-supported robot, is described. This robot was designed to perform complex three-dimensional (3D) movements and to navigate through metallic structures using a 'caterpillar'concept.

Because of the highly reliable results, a simple control running at a high speed, a permanent magnetic adhesion mechanism, a tracked locomotion mechanism are the components used to build the wall-climbing robot in this paper. As will be seen, the magnetic system is of great importance in a wall-climbing robot with a permanent magnetic adhesion mechanism because it provides reliable attraction and ensures the safety of the robot and its surroundings.

This paper is organized as follows: first, the architecture of the wall-climbing robot is briefly discussed; then the static and dynamic force of the wall-climbing robot is analysed; next, the design of the magnetic system is discussed and analysed; and finally, applications of the magnetic system and experiments are presented.

\section{ARCHITECTURE OF THE PROPOSED WALL-CLIMBING ROBOT}

It is well known that some animals can walk on walls safely and efficiently, for example, geckos and flies. Engineers working on wall-climbing robots were interested to know how these animals reliably stick to walls. Recent studies have proved that it is the physical structure of geckos' feet that allows them to stick to walls.

The physical structure of a gecko's foot is shown in Figure 1 (Autumn et al. 2000). A gecko's foot, which could lift a $60-\mathrm{lb}$ child, may have more than 1.5 million special hairs. Each hair can have from 100 to 1000 microscopic branches, like a tree. Every branch has a small pad on its tip. When a gecko takes a step, it spreads its toes. The foot is placed on the surface in a rolling fashion. Pads at the tip of each hair contact the surface and slide slightly backward. This motion brings molecules from the gecko's feet closer to molecules from the surface. If the positive side of a gecko's molecule is away from the molecule of the surface, as it approaches, the electrons in the molecule of the surface will reposition so that its positive side will be towards the gecko's molecule. For this brief moment, the negative side of the gecko's molecule faces the positive side of the molecule of the surface. The two molecules attract and stick together. They stay together because their electrons now move in unison, constantly maintaining an opposite polarity. To release its foot, a gecko simply moves it slightly. This changes the angle of each hair in relation to the surface; the attraction is broken, effortlessly. The gecko may have 6.5 million foot hairs. If all these hairs were stuck at the same time, the gecko could lift a 260-lb man.

Based on the gecko's physical behaviour, the adhesion mechanism for the robot can be designed. Although a 
wall-climbing robot must possess adequate strength to safely adhere to the wall, minimum effort is necessary in order to efficiently move on the wall. Inspired by this thought, a tracked locomotion, with small magnetic units attached on the top of the roller chains, was developed so that approximately 20 magnetic units can provide enough strength to make the robot affix itself firmly to the wall. When the robot moves on the wall, it needs to move only the first upper or lower magnetic unit to minimize the effort.

Another problem with tracked locomotion is that if there are some obstacles, such as welding seams on the oil tanks, which can make the robot lose its balance, the robot may fall off the wall. To prevent this from happening, the behaviour of a gecko's tail was analysed. The function of the tail is to keep the body in balance while walking or moving. Therefore, we designed a special anti-toppling system to balance the proposed wall-climbing robot's body. When the robot moves upward or downward, the anti-toppling system provides extra torque to push the first upper magnetic unit to stick to the wall.

On the basis of the above discussion, the mechanical architecture of the proposed wall-climbing robot is shown in Figure 2. It includes the frame of the body, the servomotors and transmission system, the tracked locomotion system, the permanent magnetic adhesion system and the anti-toppling system. Each track consists of a roller chain, two sprockets and evenly arranged permanent magnetic units. When the robot moves, a certain number of units are always in good contact with the surface, which enable the robot to maintain contact reliably. The servomotors and the transmission system provide driving power to the roller chain. The anti-toppling system ensures that the robot safely moves on the surface (up to $10 \mathrm{~mm}$ fluctuation). This paper presents in detail the design of the magnetic circuit system.

\section{STATIC AND DYNAMIC FORCE ANALYSIS OF THE WALL-CLIMBING ROBOT}

The static and dynamic force analysis of the wall-climbing robot is important to meet the requirements of the magnetic adhesion mechanism. The force analysis is undertaken under different situations while the robot moves on the wall. To move safely or to stay on a flat surface, the wall-climbing robot needs to fulfil some force constraints. Force analysis includes both static and dynamic parts.

\section{Static force analysis}

When the robot remains on the vertical surface, it bears forces such as gravity, the attractive force generated by the permanent magnetic units, the supporting force of the surface and the friction between the tracks and the surface, which can be denoted by $G, F_{m i}, N_{i}$ and $F_{f}$, respectively. There are three different cases for the static force analysis

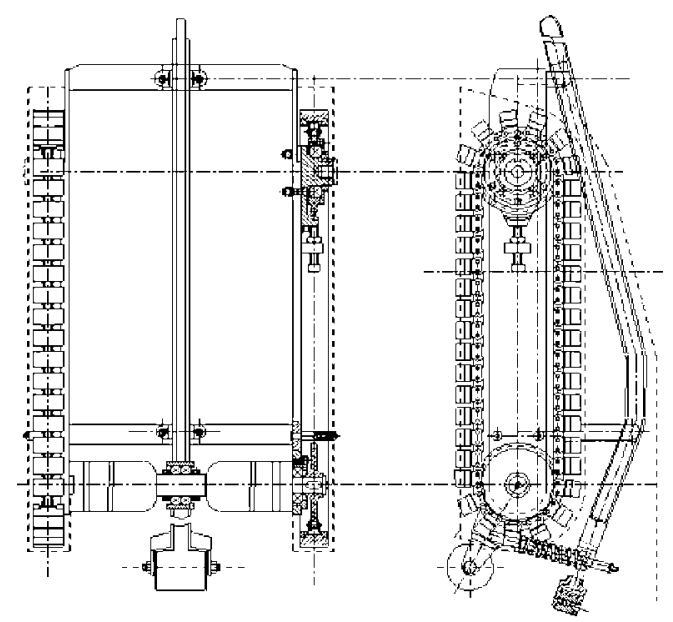

Figure 2 Mechanical architecture of the robot.

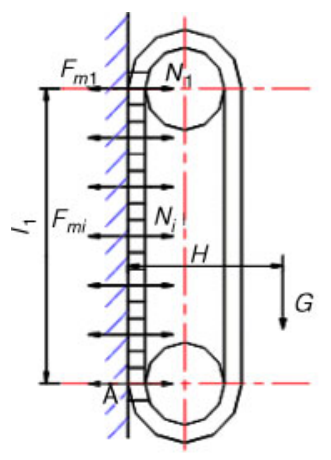

A. Robot rotating about $A$

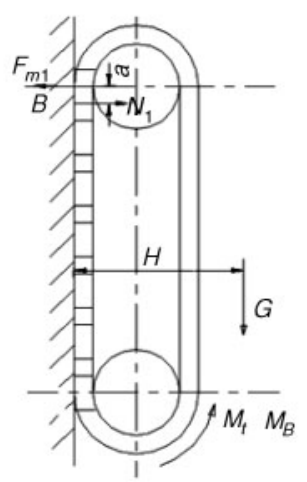

C. Tracks rolling down

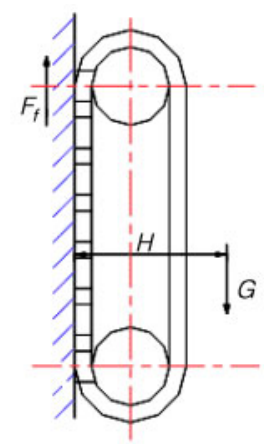

B. Robot slipping down

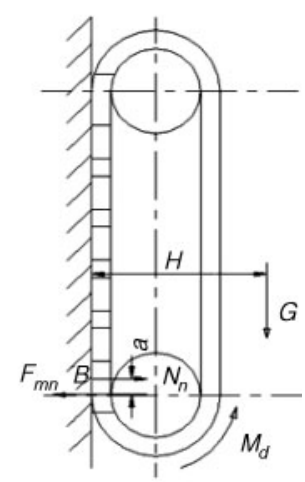

D. Robot moving up
Figure 3 Forces and torques under different situations.

when the robot maintains a static position on the vertical wall.

\section{Rotating about point $A$}

If the robot rotates about point $A$, but still maintains its balance, which is shown in Figure 3(A), we have

$$
\sum_{i=1}^{n} F_{m i}=\sum_{i=1}^{n} N_{i} .
$$

Because roller chains in the track are connected by a number of small joints, torque at point A cannot be transmitted 
by the roller chains. If the robot rotates about point $A$, the first upper magnetic unit will be lifted. If point $A$ is chosen as the rotation fulcrum, the result at point $\mathrm{A}$, as given by the torque balance equation, is

$$
\sum M_{A}=\left(F_{m 1}-N_{1}\right) l_{1}-G H / 2=0,
$$

where $l_{1}$ is the distance between the first upper magnetic unit and point $\mathrm{A}$ and $H$ is the distance between the surface and the mass centre of the robot.

From Equation (2), we have

$$
F_{m 1}=\frac{G H}{2 l_{1}}+N_{1} .
$$

If the first upper magnetic unit is attracted to the surface, we have

$$
N_{1} \geq 0 .
$$

So, Equation (3) can be rewritten as

$$
F_{m 1} \geq \frac{G H}{2 l_{1}} \text {. }
$$

Therefore, each permanent magnetic unit should fulfil Equation (5) to make the robot attach itself to the surface.

\section{Slipping down from the wall}

The robot staying on the surface will slip down if the force of the attraction is small, as shown in Figure 3(B). To overcome this problem, we impose one constraint

$$
2 F_{f} \geq G \text {. }
$$

If the friction coefficient is $\mu$, the result is

$$
F_{f}=\sum_{i=1}^{n} N_{i} \mu=\sum_{i=1}^{n} F_{m i} \mu=n F_{m} \mu .
$$

Then, according to Equations (6) and (7),

$$
F_{m} \geq \frac{G}{2 n \mu} \text {. }
$$

From Equations (5) and (8), it can be seen that the attractive force of each unit is given by

$$
F_{m} \geq \max \left\{\frac{G H}{2 l_{1}}, \frac{G}{2 n \mu}\right\} .
$$

\section{Tracks rotating on the wall}

When the robot maintains static status on the surface, provided there is no brake torque given by the motors, the tracks will rotate, as shown in Figure 3(C). By choosing point $\mathrm{B}$ as the rotation fulcrum, and assuming that the brake torque and the torque given by the transmission are $M_{b}$ and $M_{t}$, respectively, we have

$$
M_{B}=M_{b}+M_{t}+F_{m 1} a-G H / 2=0,
$$

that is,

$$
M_{\mathrm{b}}+M_{\mathrm{t}}=G H / 2-F_{m 1} a,
$$

where $a$ is the distance between $N_{1}$ and $F_{m 1}$.

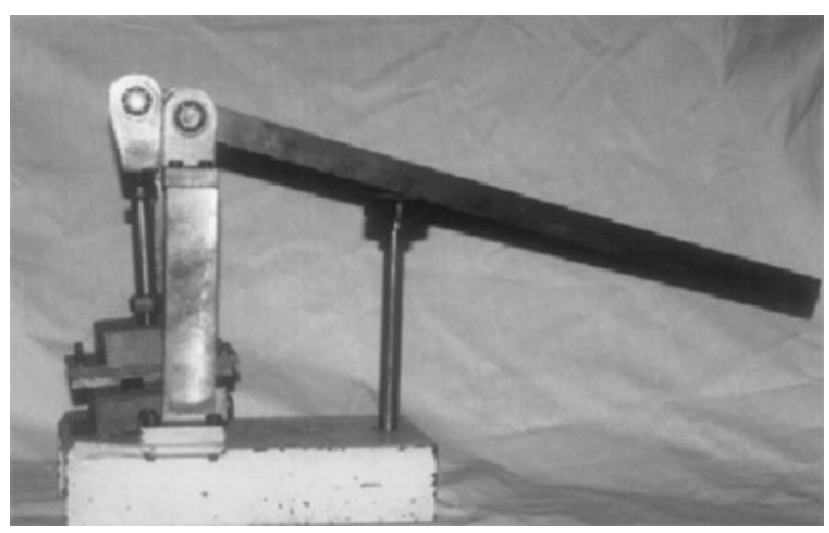

Figure 4 Lever equipment for the experiment.

\section{Dynamic force analysis}

There are two cases for dynamic force analysis when the robot moves on the wall.

\section{Robot crawling upward}

When the robot moves upward, the torque provided by the motors, $M_{d}$, should be large enough to drive the tracks. As shown in Figure 3(D), the choice of point $B$ as the rotation fulcrum results in

$$
M_{d}-F_{m n} a-G H / 2=0,
$$

that is,

$$
M_{d}=F_{m n} a+G H / 2,
$$

where $a$ is the distance between $N_{n}$ and $F_{m n}$.

\section{Robot crawling downward}

When the robot moves downward, as shown in Figure 3(C), the choice of point $\mathrm{B}$ as the rotation fulcrum results in

$$
M_{B}=M_{t}+F_{m 1} a+M_{d}-G H / 2=0,
$$

that is,

$$
M_{d}=G H / 2-M_{t}-F_{m 1} a .
$$

Therefore, the brake torque and the driving torque of motors can be calculated, by using Equations (11), (13) and (15):

$$
M_{b} \geq G H / 2-F_{m 1} a-M_{t},
$$

$$
M_{d}=\max \left\{\left(G H / 2-M_{t}-F_{m 1} a\right),\left(F_{m n} a+G H / 2\right)\right\} .
$$

\section{DESIGN OF THE MAGNETIC CIRCUIT SYSTEM}

To design the permanent magnetic unit, two problems needed to be solved. One was the selection of a suitable magnetic material and the other was the design structure of this unit. As shown in Figure 4, a lever equipment was built to facilitate the measurement of the permanent magnetic unit. 


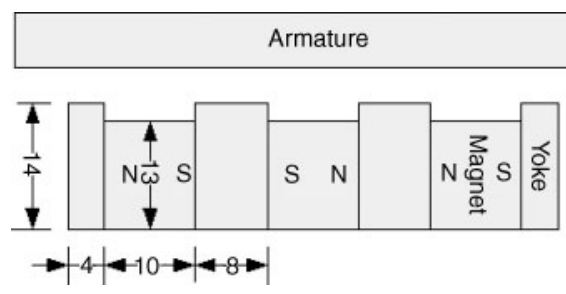

Figure 5 Type A structure of the magnetic unit.

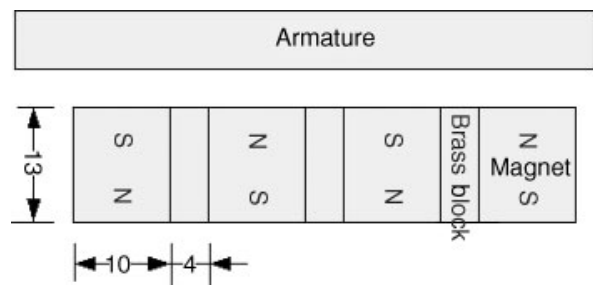

Figure 6 Type B structure of the magnetic unit.

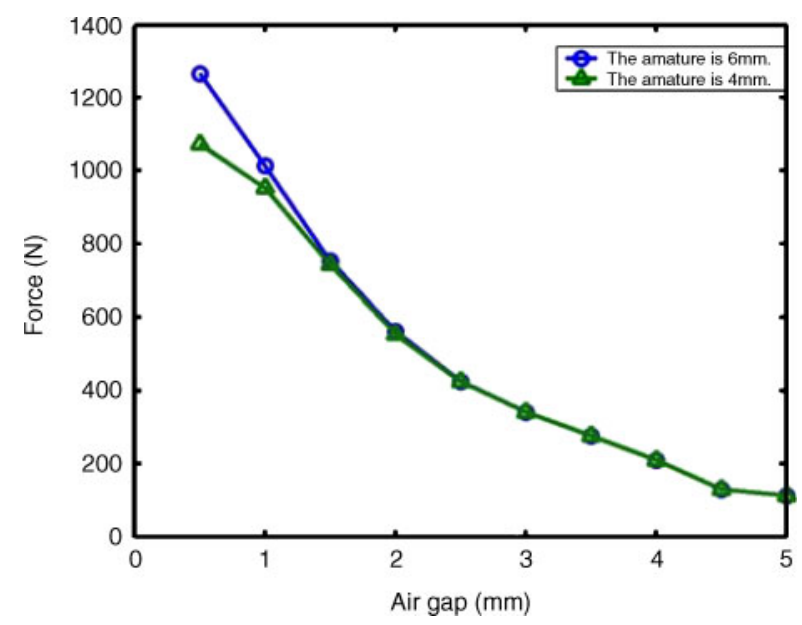

Figure 7 Influence of thickness of the armature.

\section{Selection of magnetic material}

A permanent high performance magnet with the standard ingredients $\mathrm{Nd}_{15} \mathrm{Fe}_{77} \mathrm{~B}_{8}$ was adopted. The parameters of this material are listed in Table 1.

\section{Magnetic unit's structure}

The structure of the magnetic unit was critical because an efficient structure can have small volume but large attraction force.

Two kinds of structures were designed for the magnetic circuit system, which are shown in Figures 5 and 6 . Both of these are considered efficient.

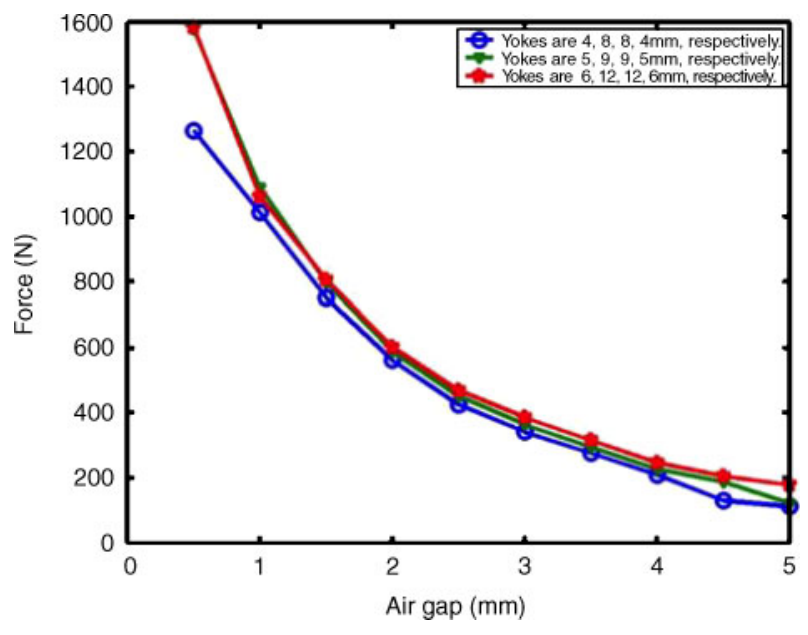

Figure 8 Influence of thickness of the yokes.

\section{Analysis of type A structure}

To test type A structure, three different experiments were performed.

\section{Influence of thickness of the armature}

In Figure 7, when the thickness of the armature is $6 \mathrm{~mm}$, the curve with circle markers indicates the relationship between the forces generated by type A structure and the air gap. When the thickness of the armature is $4 \mathrm{~mm}$, the curve with triangle markers shows the relationship between the two.

\section{Influence of thickness of the yokes}

Assume that the thickness of the armature is $6 \mathrm{~mm}$. In Figure 8 , the curve with circle markers shows the relationship between the forces generated by the type A structure and the air gap, when the thickness of the yokes is 4,8 , 8 and $4 \mathrm{~mm}$, respectively; the curve with triangle markers shows the relationship when the thickness of the yokes is $5,9,9$ and $5 \mathrm{~mm}$, respectively; the curve with pentagram markers shows the relationship when the thickness of the yokes is $6,12,12$ and $6 \mathrm{~mm}$, respectively.

\section{Influence of the difference in heights of the magnets and the yokes}

In Figure 9, the curve with circle markers shows the relationship between the forces generated by type A structure and the air gap, when the difference in the height of the magnets and the yokes is $1 \mathrm{~mm}$; the curve with triangle markers shows the relationship when there is no difference in their heights.

Table 1 Main parameters of $\mathrm{Nd}_{15} \mathbf{F e}_{77} \mathbf{B}_{8}$

\begin{tabular}{lllll}
\hline$B_{\mathrm{r}}(T)$ & $H_{\mathrm{CB}}(\mathrm{KA} / \mathrm{m})$ & $H_{\mathrm{ci}}(\mathrm{KA} / \mathrm{m})$ & $H B\left(\mathrm{KJ} / \mathrm{m}^{3}\right)$ & Rate of hysteresis $\left(\mu_{\mathrm{rec}}\right)$ \\
\hline $1.06-1.16$ & $800-880$ & $>1360$ & $224-248$ & $1.05-1.10$ \\
\hline
\end{tabular}




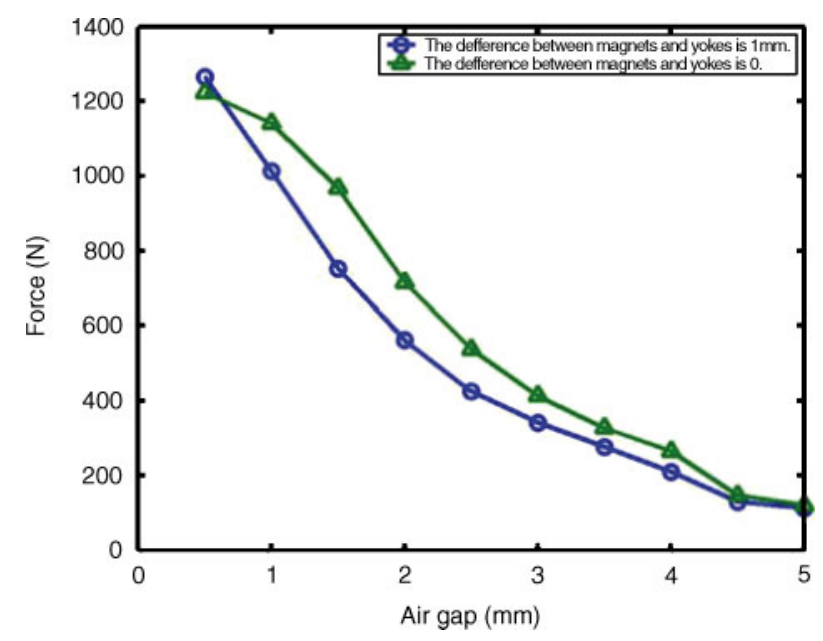

Figure 9 Influence of difference of the height of the magnets and the yokes.

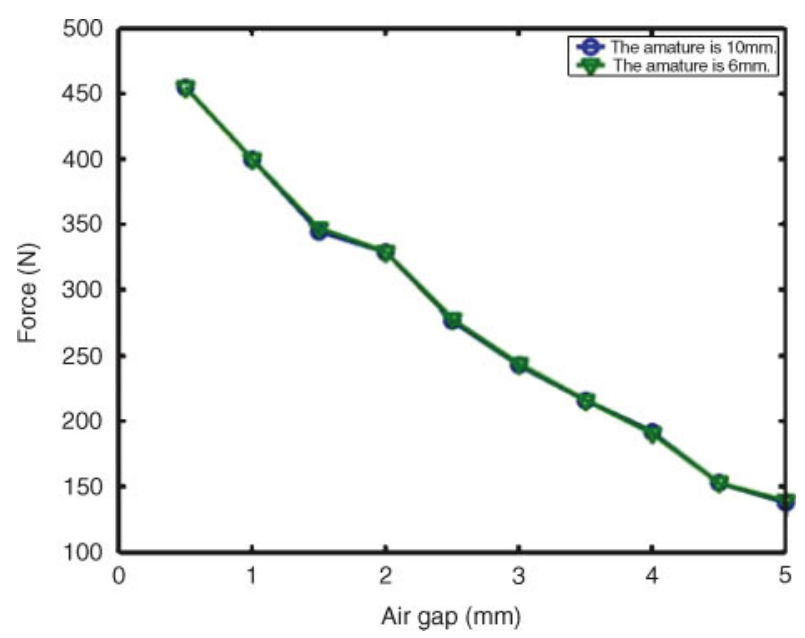

Figure 10 Influence of thickness of the armature.

\section{Analysis of type B structure}

To test type B structure, two different experiments were performed.

\section{Influence of thickness of the armature}

Assume that the thickness of the brass blocks is $4 \mathrm{~mm}$. In Figure 10, the curve with circle markers shows the relationship between the forces generated by type B structure and the air gap when the thickness of the armature is $10 \mathrm{~mm}$; the curve with triangle markers shows the relationship when the thickness of the armature is $6 \mathrm{~mm}$.

\section{Influence of the thickness of the brass blocks}

Assume that the thickness of the armature is $10 \mathrm{~mm}$. In Figure 11, the curve with circle markers shows the relationship between the forces generated by type B structure and the air gap when the thickness of the brass blocks is $4 \mathrm{~mm}$; the curve with triangle markers shows the relationship when the thickness of the brass blocks is $2 \mathrm{~mm}$.

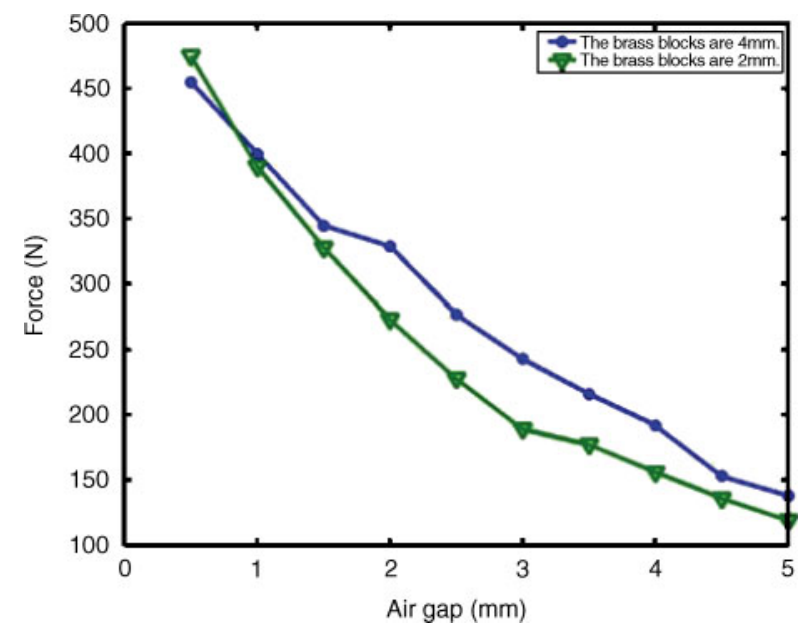

Figure 11 Influence of thickness of the brass blocks.

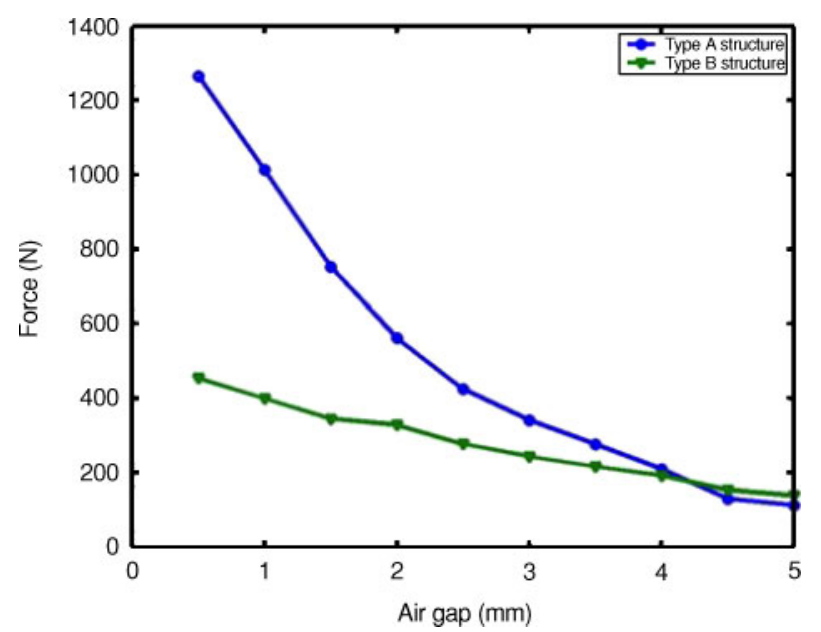

Figure 12 Comparison of the two types of structures.

\section{COMPARISON OF THE TWO STRUCTURES}

The two structures are compared in Figure 12. The curve with circle markers is for type A structure, and the curve with triangle markers is for type B structure. If the air gap is less than $4.25 \mathrm{~mm}$, type $\mathrm{A}$ structure generates a stronger force than do type B structure. However, the force generated by type A structure decreases dramatically with an increase in the air gap.

\section{APPLICATIONS AND EXPERIMENTS}

In two different wall-climbing robots, two types of magnetic units have been used to inspect water tubes in power stations and in oil tanks.

In the first application, there is one limitation regarding the volume of the robot. By the comparison and discussion in the above section, it is clear that type A structure has high efficiency. Therefore, type A structure is chosen. For designing the wall-climbing robot, the number of permanent magnetic units was taken as 84 . Among them, 


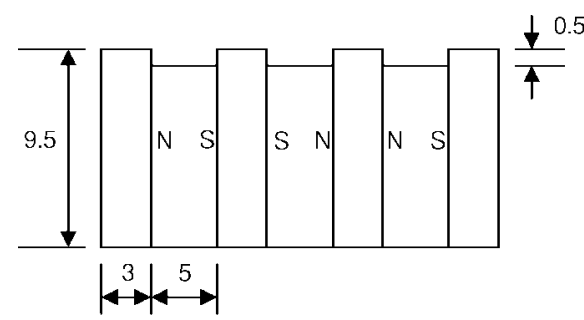

Figure 13 Type A structure for the first application.

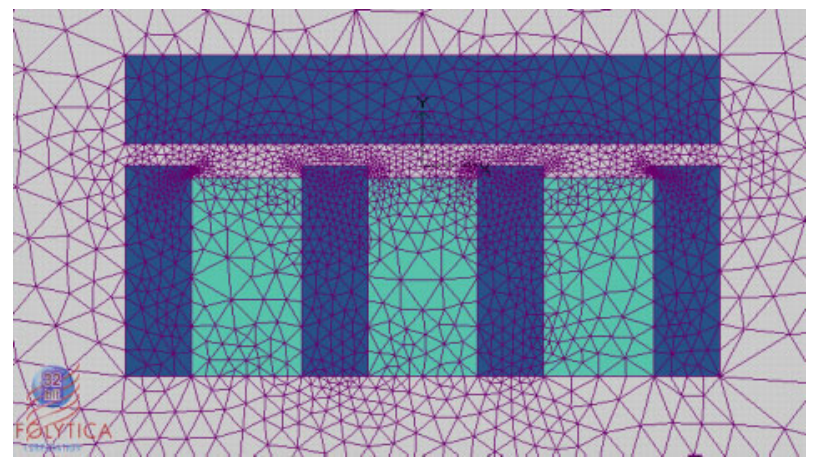

Figure 14 The mesh of the models.

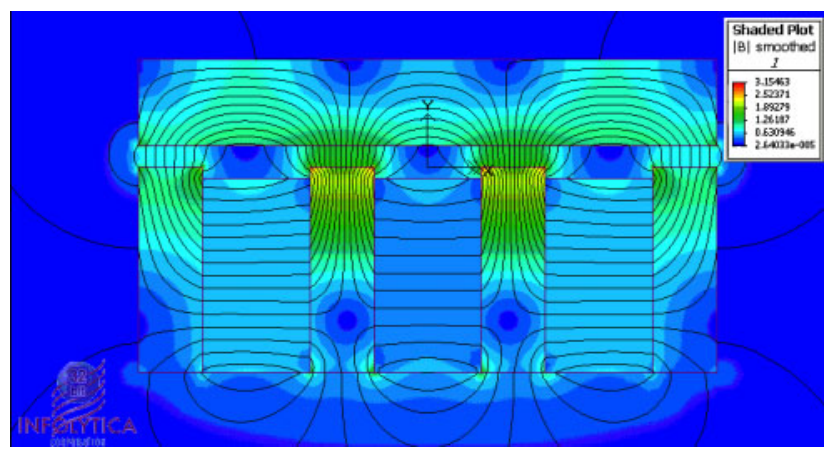

Figure 15 The magnetic flux and flux density.

there are 30 units being attracted on the surface. The force requirement for one unit is given by

$$
19.6 \mathrm{~N} \leq F_{m} \leq 6533 \mathrm{~N} .
$$

Considering safe tolerances and the current technology, we have

$$
150 \mathrm{~N} \leq F_{m} \leq 600 \mathrm{~N} .
$$

The attractive force of each permanent magnetic unit must be more than the minimum value, so that the robot can safely attach itself to the wall. Furthermore, the attractive force must be less than the maximum value, otherwise more powerful motors will be required to drive the robot.

The thickness of the water tube is around $4-6 \mathrm{~mm}$, and the width of the air gap is around $0.5-1 \mathrm{~mm}$. The parameters for this magnetic unit are shown in Figure 13. To verify the forces generated by type A structure, the finite element method (FEM) was employed to analyse the two-dimensional (2D) static magnetic field. The mesh of

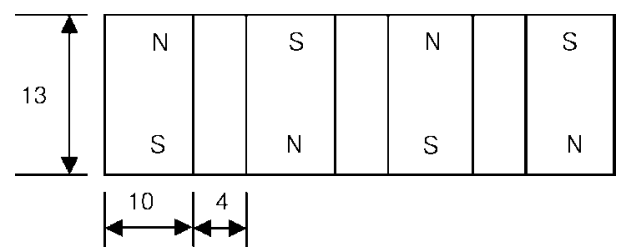

Figure 16 Type B structure for the second application.

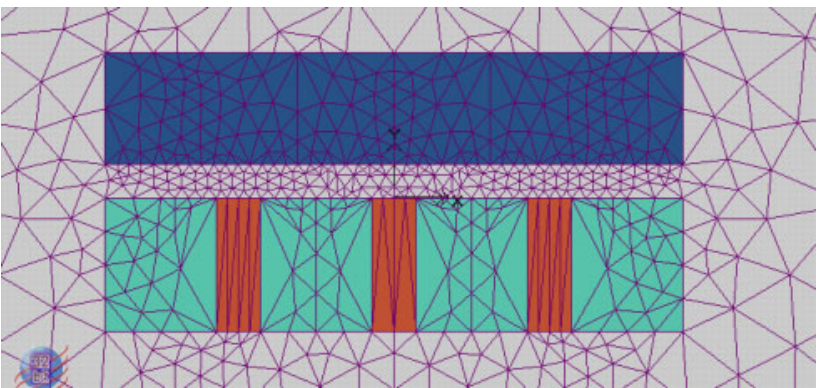

Figure 17 The mesh of the models.

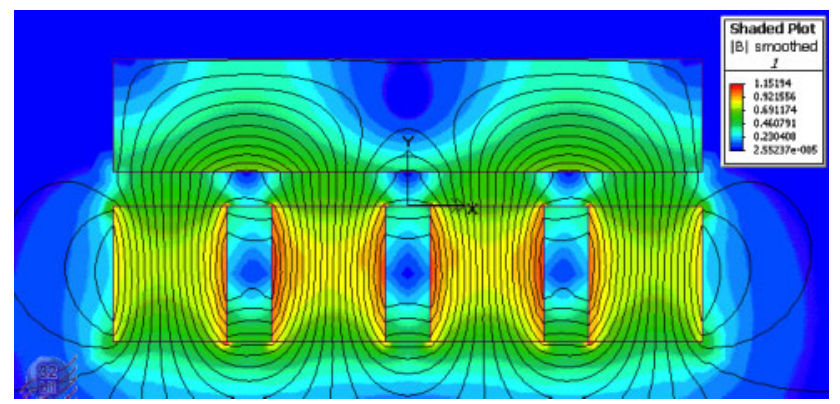

Figure 18 The magnetic flux and flux density.

the models is shown in Figure 14, and the magnetic flux and the magnetic flux density are shown in Figure 15.

For calculating the force, the thickness of the water tube was taken as $6 \mathrm{~mm}$ and the width of the air gap was taken as $1 \mathrm{~mm}$. The force generated by type A structure was more than $320 \mathrm{~N}$, which fulfils the requirements.

In the second application, the thickness of oil tanks was around $6-10 \mathrm{~mm}$ and the width of the air gap was around 2.5-3.0 mm, so type B structure was chosen. Considering other constraints, in the design of the wall-climbing robot, the number of permanent magnetic units was taken as 44 . Among them, there were 18 units being attracted on the surface. The force required by one unit is given by

$$
15 \mathrm{~N} \leq F_{m} \leq 6533 \mathrm{~N} \text {. }
$$

Considering safe tolerances and the current technology, we have

$$
140 \mathrm{~N} \leq F_{m} \leq 600 \mathrm{~N} .
$$

Finally, different parameters for this unit are presented in Figure 16. To verify the force generated by type B structure, FEM was used to analyse the 2D static magnetic field. The mesh of the models is shown in Figure 17, and the magnetic flux and the magnetic flux density are shown in Figure 18. 


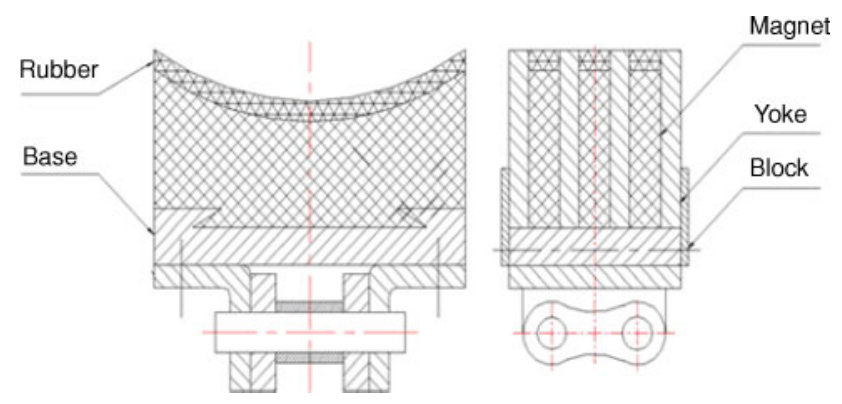

Figure 19 The magnetic unit using type A structure.
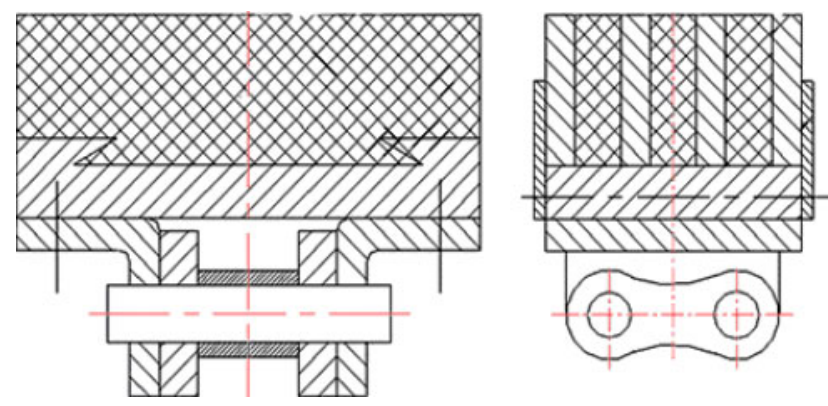

Figure 20 The magnetic unit using type B structure.

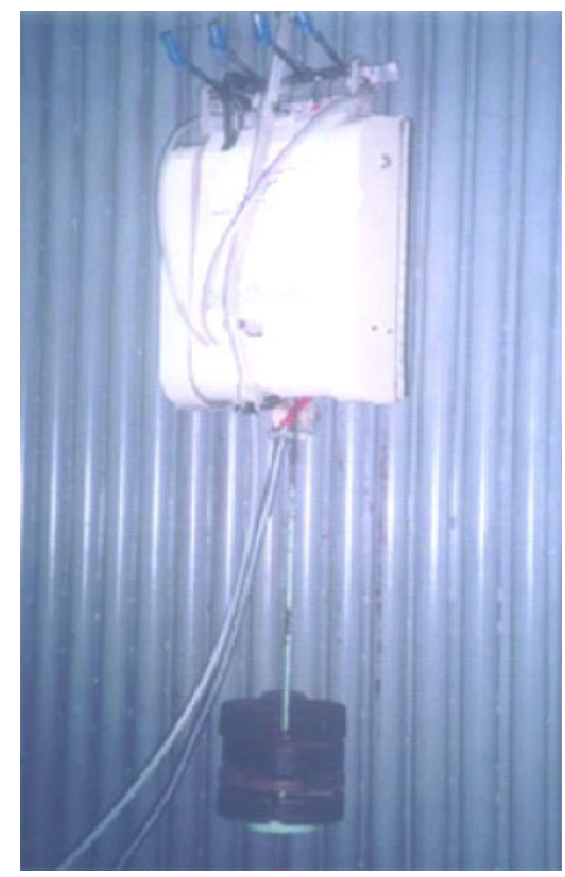

Figure 21 Wall-climbing robot for inspection of a water tube.

For calculating the force, the thickness of the oil tanks was taken as $10 \mathrm{~mm}$ and the width of the air gap was taken as $3 \mathrm{~mm}$. The force generated by type B structure was more than $150 \mathrm{~N}$, which also fulfils the requirements.

Finally, two different magnetic units were designed, which are shown in Figures 19 and 20.

Wall-climbing robot for inspecting a pater tube. In this case, the size of the magnetic unit is $30 \mathrm{~mm} \times 27 \mathrm{~mm}$ $\times 20 \mathrm{~mm}$, the weight is $1.46 \mathrm{~N}$, the attractive force generated by one unit is $320 \mathrm{~N}$ at $1 \mathrm{~mm}$ of the air gap and

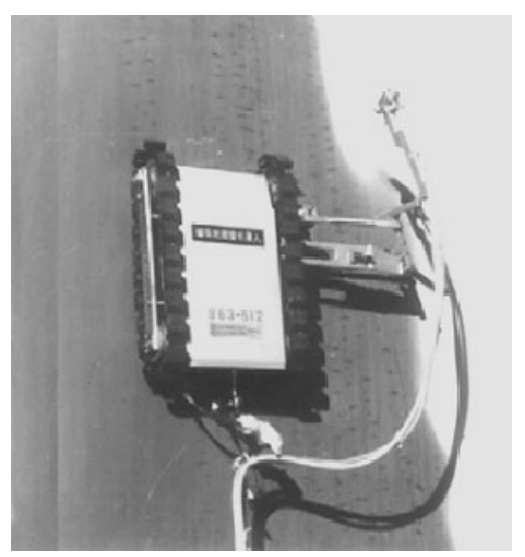

Figure 22 Wall-climbing robot for oil tank inspections.

the force generated by the whole magnetic system is more than $9600 \mathrm{~N}$. The robot is shown in Figure 21. This robot can move on the wall very reliably and safely with payloads more than $400 \mathrm{~N}$.

Wall-climbing robot for inspecting oil tanks. In this case, the size of the permanent magnetic unit is $55 \mathrm{~mm} \times$ $52 \mathrm{~mm} \times 28 \mathrm{~mm}$, the weight is $3.5 \mathrm{~N}$, the attractive force generated by one unit is $150 \mathrm{~N}$ at $3 \mathrm{~mm}$ of the air gap and the force generated by the whole magnetic system is more than $2700 \mathrm{~N}$. The robot is shown in Figure 22. This robot can also move on the wall very reliably and safely with payloads more than $350 \mathrm{~N}$.

\section{CONCLUSIONS}

This paper presented the design of the magnetic system for the wall-climbing robot with a magnetic adhesion mechanism and a tracked-driven locomotion mechanism. Based on the behaviour of gecko lizards, the architecture of the robot was designed and built, including the structure of the adhesion mechanism, the mechanical architecture and the anti-toppling mechanism, which is important to ensure that the robot can work safely and efficiently on a vertical wall. The static and dynamic force analysis of the robot was also undertaken under different situations in this work. Based on the discussion and analysis of two different types of structures of the permanent magnetic unit, their application limitations were discussed. If the air gap was smaller than $3.5 \mathrm{~mm}$, type A structure had better performance. However, if the air gap was larger than $3.5 \mathrm{~mm}$, type B structure had better performance. The selection of the structures depends on their applications. The finite element method was also used to verify the above results. Based on two different applications, two wall-climbing robots with two different magnetic systems were built and tested. Experimentally, both robots were considered reliable and safe, and could carry loads more than $350 \mathrm{~N}$, which further confirmed the design of permanent magnetic system. 


\section{REFERENCES}

Abderrahim M, Balaguer C, Gimenez A, Pastor JM, Padron VM. 1999. ROMA: A climbing robot for inspection operations. In IEEE International Conference on Robotics and Automation, pp. 2303-8.

Almonacid M, Saltaren RJ, Aracil R, Reinoso O. 2003. Motion planning of a climbing parallel robot. IEEE Trans Robot Autom, 19(3):485-9.

Amano H, Osuka K, Tarn TJ. 2001. Development of vertically moving robot with gripping handrails for fire fighting. In 2001 IEEE/RSF International Conference on Intelligent Robots and Systems, pp. 661-7.

Autumn K, Chang W-P, Fearing R, Hsieh T, Kenny T, Liang L, Zesch W, Full RJ. 2000. Adhesive force of a single gecko foot-hair. Nature, 405:681-5.

Bretl T, Rock S, Latombe JC. 2003. Motion planning for a three-limbed climbing robot in vertical natural terrain. In IEEE International Conference on Robotics and Automation, pp. 2946-53.

Briones L, Bustamante P, Serna MA. 1994. Wall-climbing robot for inspection in nuclear power plants. In IEEE International Conference on Robotics and Automation, pp. 1409-14.

Griecho JC, Prieto M, Armada M, Gonzales SP. 1998. A six-legged climbing robot for high payloads. In IEEE International Conference on Control Applications, pp. 446-50.

Kang T, Kim H, Son T, Choi H. 2003. Design of quadruped walking and climbing robot. In IEEE/RSJ International Conference on Intelligent Robots and Systems, pp. 619-24.

Luk BL, Collie AA, Billingsley J. 1999. Robug II: An intelligent wall-climbing robot. In IEEE International Conference on Robotics and Automation, pp. 2342-7.

Nishi A, Miyagi H. 1994. Mechanism and control of propeller type wall-climbing robot. In IEEE/RSJ International Conference on Intelligent Robots and Systems, pp. 1724-9.
Nishi A, Wakasugi Y, Watanabe K. 1986. Design of a robot capable of moving on a vertical wall. Adv Robot, 1:33-45.

Savall J, Avello A, Briones L. 1999. Two compact robots for remote inspection of hazardous areas in nuclear power plants. In IEEE International Conference on Robotics and Automation, pp. 1993-8.

Schmid D, Macute B, Merettig H. 1998. Tracked robot go up to wall. In CLAWAR 98, pp. 23-40.

Shen W, Gu J, Shen Y. 2005. Proposed wall-climbing robot with permanent magnetic tracks for inspecting oil tanks. In IEEE International Conference of Mechatronic and Automation, pp. 2072-8.

Sitti M, Fearing RS. 2003. Synthetic gecko foot-hair micro/nano-structures for future wall-climbing robots. In IEEE International Conference on Robotics and Automation, pp. 1164-70.

Tummala RL, et al. 2002. Climbing the walls [robots]. IEEE Robot Autom Mag, 9(4): 10-9.

Wang Y, Liu S, Xu D, Zhao Y, Shao H, Gao X. 1999. Development and application of wall-climbing robots. In IEEE International Conference on Robotics and Automation, pp. 1207-12.

White T, Hewer N, Luk N, Hazel J. 1998. The design and operational performance of a climbing robot used for weld inspection in hazardous environments. In IEEE International Conference on Control Applications, pp. 451-5.

Xiao J, Xiao J, Xi N. 2003. Minimal power control of a miniature climbing robot. In IEEE/ASME International Conference on Advanced Intelligent Mechatronics, pp. 616-21.

Zhang HX, Zhang JW, Zong GH. 2004a. Requirements of glass cleaning and development of climbing robot systems. In IEEE International Conference on Intelligent Mechatronics and Automation, pp. 101-106.

Zhang, HX, Zhang JW, Zong GH. 2004b. A novel approach to pneumatic position servo control of a glass wall cleaning robot. In IEEE / RSJ International Conference on Intelligent Robots and Systems, pp. 467-72. 

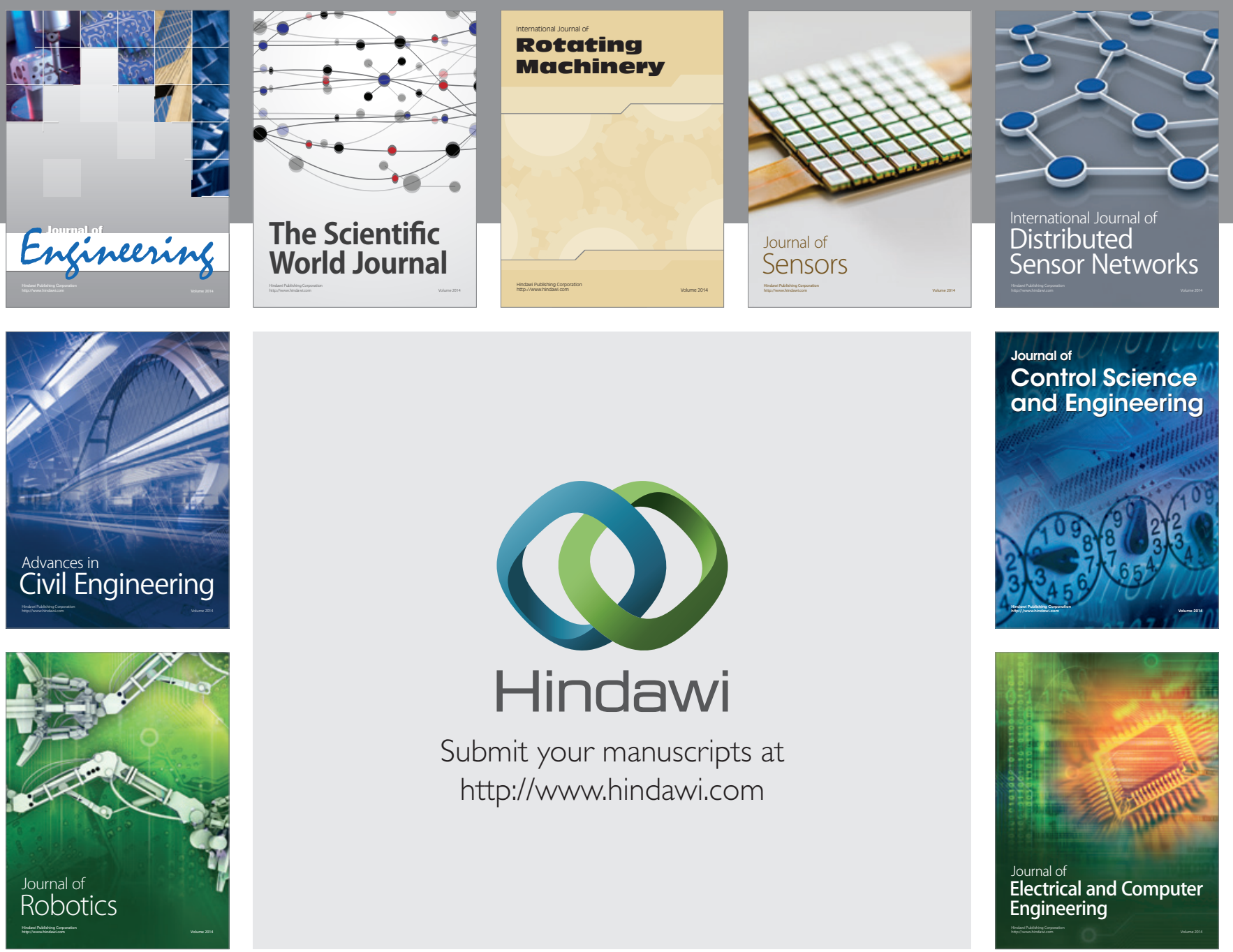

Submit your manuscripts at

http://www.hindawi.com
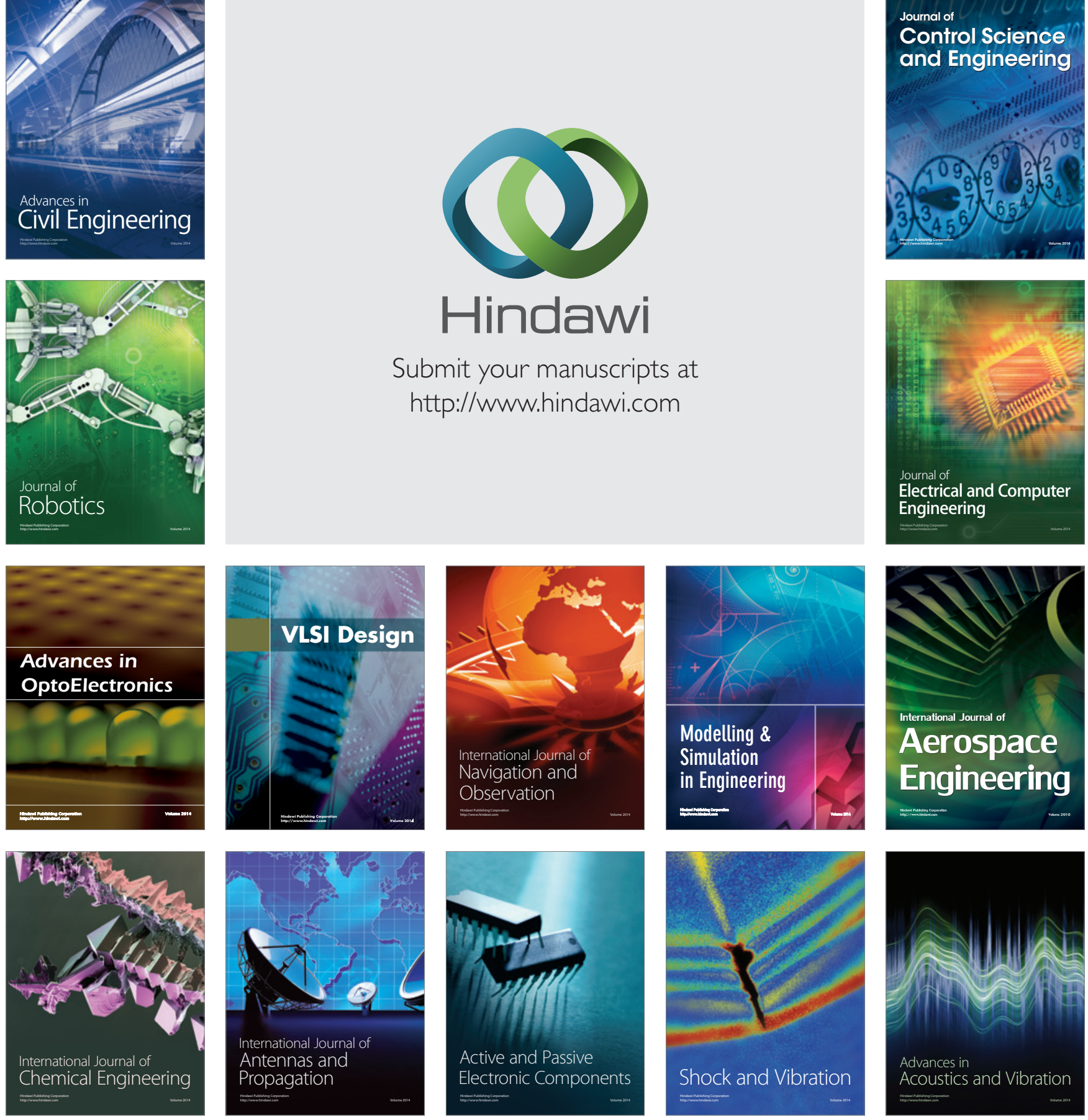